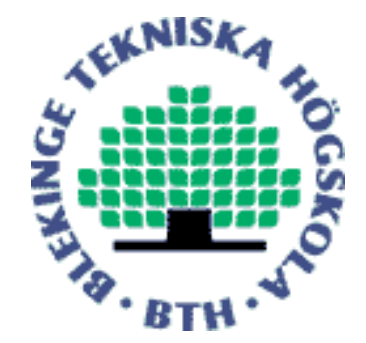

Copyright (C) 2011 IEEE.

Citation for the published paper:

Implementation and evaluation of NIST Biometric Image Software for fingerprint recognition

Sainath Maddala, Sreekanth Rao Tangellapally, Josef Ström Bartunek, Mikael Nilsson

ISSNIP Biosignals and Biorobotics Conference: Biosignals and Robotics for Better and Safer Living, BRC

2011 Vitoria

This material is posted here with permission of the IEEE. Such permission of the IEEE does not in any way imply IEEE endorsement of any of BTH's products or services Internal or personal use of this material is permitted. However, permission to reprint/republish this material for advertising or promotional purposes or for creating new collective works for resale or redistribution must be obtained from the IEEE by sending a blank email message to pubs-permissions@iee.org.

By choosing to view this document, you agree to all provisions of the copyright laws protecting it. 


\section{Implementation and Evaluation of NIST Biometric Image Software for Fingerprint Recognition}

\author{
Sainath Maddala, Sreekanth Rao Tangellapally \\ Graduate Student, Dept. of Signal Processing, School of \\ Engineering, Blekinge Institute of Technology, \\ SE- 371 79, Karlskrona, Sweden \\ E-mails: sainath.madhala@gmail.com, \\ sris.chinnu@gmail.com
}

\begin{abstract}
Fingerprints are rich in details which are in the form of discontinuities in ridges known as minutiae and are unique for each person. This paper describes implementation and evaluation of an existing fingerprint recognition system in MATLAB environment. The selected system is developed by National Institute of Standards and Technology (NIST) denoted as Biometric Image Software (NBIS). The NBIS source code is written in $A N S I C$ programming language. To be able to evaluate the algorithm in MATLAB a $C$ language $M E X$-files has been used. The NBIS support both minutiae extraction and minutiae matching functions that have been employed in the evaluation. The implemented system has been tested on a Fingerprint Verification Competition (FVC) database. The results are presented as Receiver Operating Characteristics (ROC) graphs.
\end{abstract}

Keywords- Biometric; NIST Fingerprint Verification; MEXfile; ROC graphs.

\section{INTRODUCTION}

\footnotetext{
"Biometrics" (bios metron = "life measurement") is defined as the automated methods of identifying or authenticating the identity of a living person based upon one or more intrinsic physical or behavioral traits. A number of biometric technologies have been developed and are being used in numerous applications such as secure identification and personal verification solutions etc. Some examples of different biometrics are fingerprints, face recognition, iris, retina scan, signature etc. "Although biometrics emerged from its extensive use in law enforcement to identify criminals (e.g., illegal aliens, security clearance for employees for sensitive jobs, fatherhood determination, forensics, and positive identification of convicts and prisoners), it is being increasingly used today to establish person recognition in a large number of civilian applications"[1]. Human Fingertips are fully formed at about seven months of fetus development and ridge configurations which form distinctive patterns. One's fingerprint is formed those patterns do not change throughout the life of an individual except due to accidents such as bruises and cuts on the fingertips. Print of these patterns is known as fingerprints [2].

This paper is written based on Master Thesis [3]. In this paper implement and evaluation of NIST Biometric Image Software for fingerprint recognition in MATLAB environment is performed. The NBIS source code is entirely written in ANSI C programming language. So $M E X$-files are
}

\author{
Josef Ström Bartůněk, Mikael Nilsson \\ Dept. of Signal Processing, School of Engineering, \\ Blekinge Institute of Technology, \\ SE- 371 79, Karlskrona, Sweden \\ E-mails: jsb@bth.se,mkn@bth.se
}

used to run $c$ programming code in MATLAB. First fingerprint image is enhanced and minutiae are extracted. The extracted minutiae are passed through the function that match two minutiae patterns and produce a match score. Genuine match and imposter match classification is performed based on the match score. Performance of the system is evaluated on various fingerprint databases and the results obtained are presented in Receiver Operating characteristics (ROC) graphs.

\section{OPERATIONS PERformed ON Fingerprint}

Minutiae from the fingerprint image can be extracted directly from the raw grayscale fingerprints. However, the extraction process is difficult and not that accurate due to the sensor noise present in the fingerprint image. The skin conditions on the fingertip also influence the overall quality of the captured fingerprints. Due to these reasons the extracted minutiae from the fingerprint may contain both real and false minutiae. To minimize extraction of false minutiae from the fingerprint image the NBIS incorporates a preprocessing and post-processing stage in the minutiae extraction procedure. Fig.1 illustrates operations performed to limit the number of extracting false minutiae from the fingerprint.

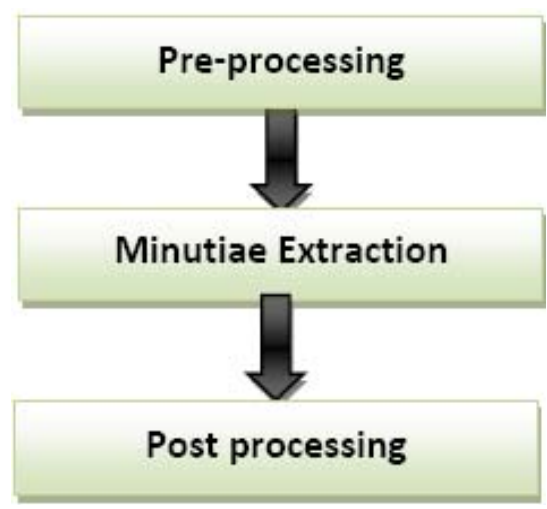

Figure 1. Flowchart representing operations performed by the NBIS before and after extracting minutiae. 


\section{A. Pre-processing}

Fingerprint images acquired with various sensors will have different levels of the dynamic range altering the image contrast. Therefore a pre-processing operation in form of contrast enhancement should be performed before extracting the minutiae from the fingerprint. The contrast enhancement can be performed with various methods. For example histogram equalization can increase the contest of the image. The function trim histtails contrast boost () developed by NIST use a histogram modulation for increasing the contrast of the fingerprint image, see Fig.2. Quality of the image can also be increased by using the filters. Low pass filter lowers the noise from the image. Band pass filter can lower undesired noise from orientations which helps to preserve true ridges [4]. Image enhancement can also be performed using Fourier transform method [5].
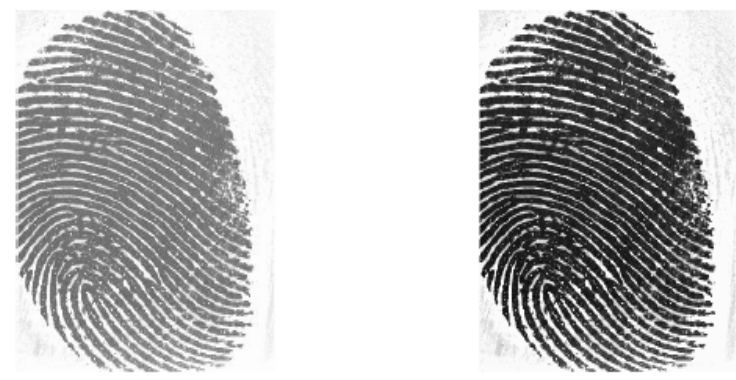

Figure 2. Fingerprint obtained after preprocessing : (a) Original image, (b) Enhanced image

\section{B. Minutiae Extraction}

Fingerprint image is a line patterns consisting of ridges, valleys, cores, deltas, pores etc as shown in Fig.3a. The most interesting patterns are ridge endings and ridge bifurcations. These patterns are used for comparing two fingerprints with each other. Fig.3b illustrates ridge ending and ridge bifurcation which plays a vital role in fingerprint matching known as minutiae. These ridge ending and ridge bifurcation do not change over time, therefore well suited for fingerprint matching. Fingerprint usually consists of 40 to 100 minutiae points.
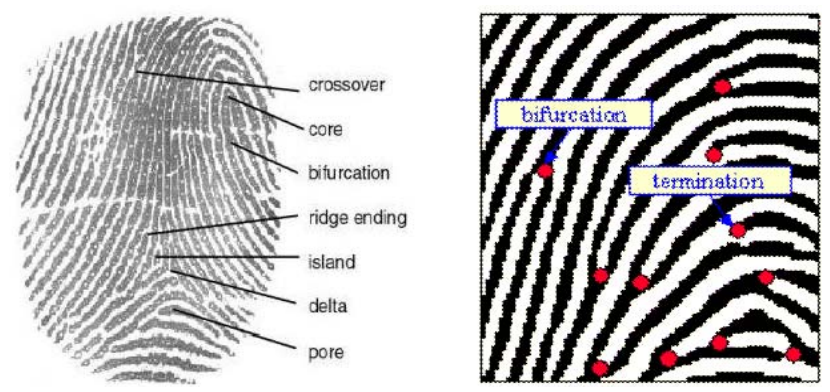

Figure 3. (a) Fingerprint image, (b) Fingerprint image with bifurcations and terminations.
The NBIS before detecting the minutiae from the fingerprint converts the grayscale image into binarized image where black pixels represents ridges and white pixels represents valleys. Based on the ridge flow direction associated with the block the pixel is assigned a binary value. For this process each pixel in the image must be analyzed for assigning white and black pixel. Then the binary image is scanned to identify ridge endings and ridge bifurcation. Pattern scanning is performed both horizontally and vertically with $2 \times 3$ pixel window size. Patterns represented in Fig.4 illustrate procedure for detecting the minutiae points in the binarized fingerprint image. Two patterns in the Fig.4 represent ridge endings and rest represents bifurcations.

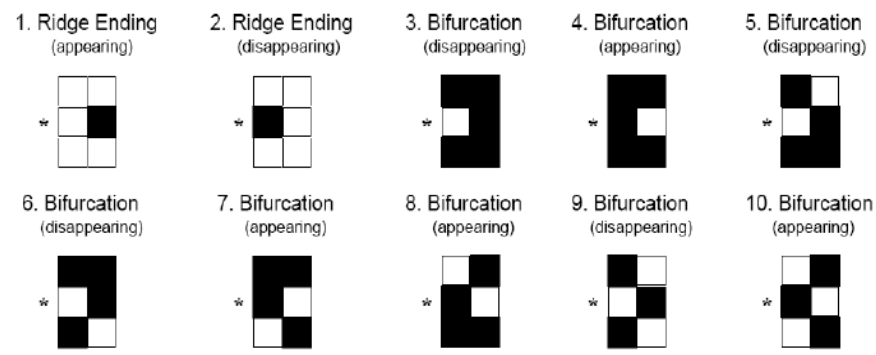

Figure 4. Pixel patterns used to detect minutiae

Minutiae detected in the fingerprint are as shown in the Fig.5 which may consist of real and false minutiae. The number of falsely detected minutiae can be minimized in post-processing stage with the help of quality factor. The algorithm developed by NIST used for minutiae detection is implemented in get_minutiae().

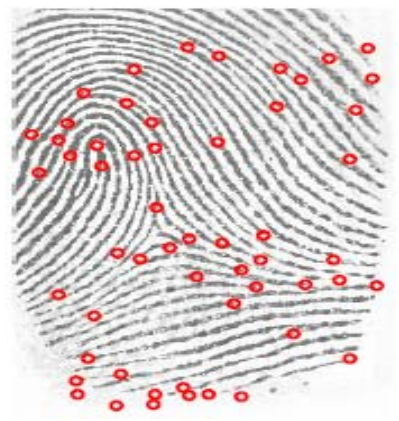

Figure 5. Minutiae obtained with NIST's method, includiong both true and false minutiae.

\section{Post processing}

Minutiae extracted from the fingerprint consist of real and to some extent of false minutiae. Various types of false minutiae are shown in Fig.6. The number of falsely detected minutiae depends upon the quality of the fingerprint. The extracted minutiae should be filtered to remove as many of the false minutiae as possible without removing real minutiae. The redundant minutiae in the fingerprint are of the form

- a) Minutiae points adjacent to each other 
- b) Minutiae near the borders

- c) Spike, break, bridge, hole as shown in the Fig.6 (a)

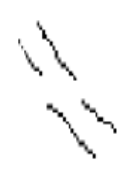

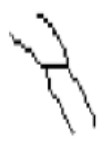

(b)

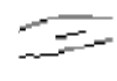

(c)

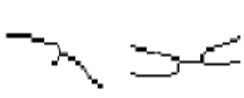

(d)

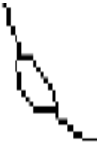

(f)
Figure 6. (a) Broken ridges, (b) Bridge, (c) Short ridge, (d) Short ridge, (e) Short Ridge, (f) Hole

These types of false minutiae may cause serious problem during matching. Removing all false minutiae separately is time taking and complex. For this reason the NBIS calculate quality of each minutia. First reliability measure is calculated based on pixel intensity statistics (mean and standard deviation) within the immediate neighborhood of the minutiae point. The size of neighborhood is set to 11 pixels. The high quality regions will cover full grayscale spectrum. Reliability, $R$, is calculated with neighborhood mean, $\mu$, and standard deviation, $\sigma$ [5]. Minutiae with lower quality correspond to false minutiae and minutiae with high quality correspond to real minutiae. The false minutiae in the fingerprint can be removed by keeping proper threshold value. The minutiae which are less than the threshold are not allowed i.e. lower quality (false minutiae) and rest of the minutiae which fulfils the threshold value are allowed i.e. high quality(real minutiae). Minutiae obtained are plotted on the fingerprint Fig.7a and minutiae with low quality are plotted as "+" and minutiae with high quality are plotted as "o" in Fig.7b.

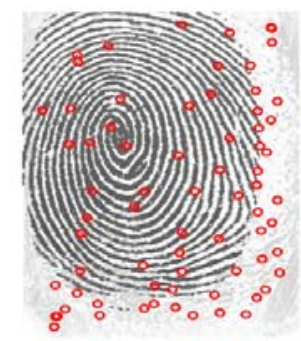

(a)

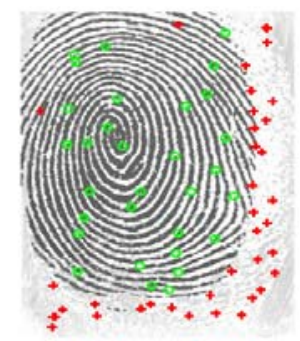

(b)
Figure 7. (a) Fingerprint image containing both false and real minutiae, (b) Fingerprint image after thresholding minutiae based on the quality, real minutiae " $o$ " and false minutiae "+".

\section{FINGERPRINT MATCHING}

BOZORTH3 is an algorithm included in NBIS that matches two minutiae patterns with each other and produces a match score. Matching between the fingerprints can be performed as one-to-one (verification) or one-to-many (identification). Bozorth3 algorithm includes three steps for fingerprint matching
- Construction of Intra-Fingerprint Minutiae Comparison Tables.

- Construction of Inter-Fingerprint Compatibility Table.

- Traverse Inter-Fingerprint Compatibility Table constructed in second step.

\section{A. Results obtained for Genuine match and Imposter match}

First minutiae tables are constructed for both probe and gallery fingerprint as shown in Fig.8. These tables are given to the minutiae matcher for generating the scores. $S(P 1, P 2)$ represents scores obtained from genuine matach where $P 1$ and $P 2$ are the fingerprints from same person. $S(G 1, G 2)$ represents scores obtained from imposter matach where $G 1$ and $G 2$ are the fingerprints from different persons. The matching score with greater value will indicate that the fingerprints are more likely from the same person.

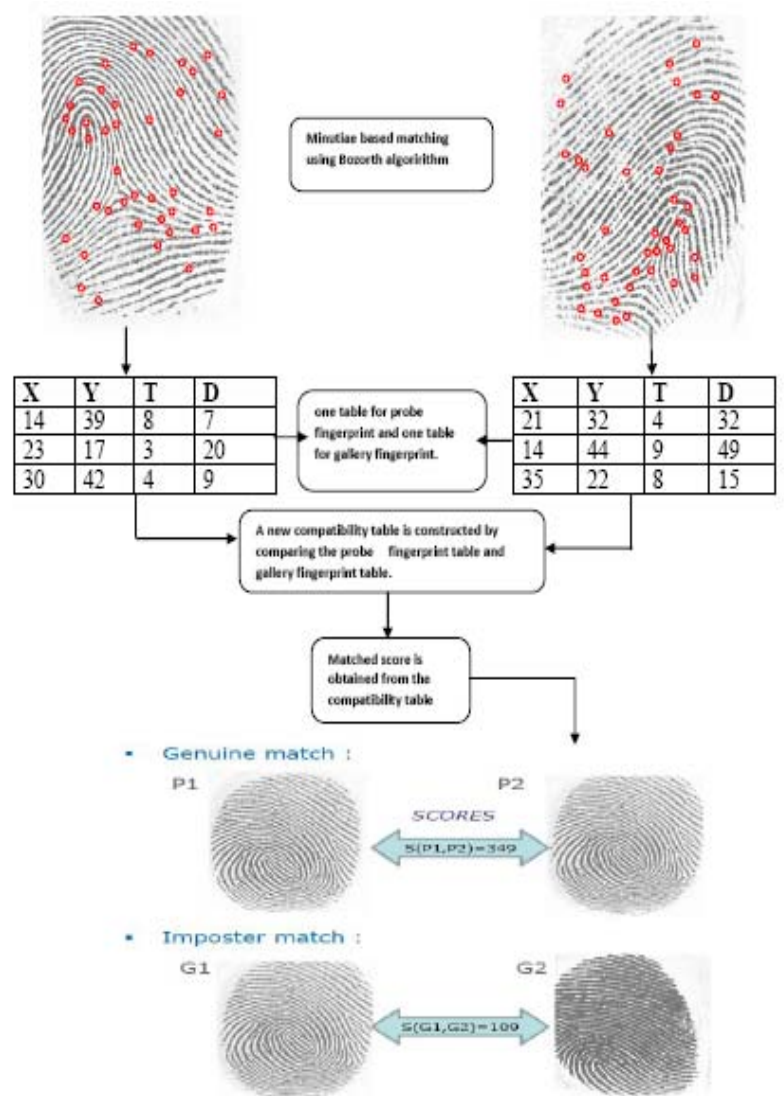

Figure 8. Flowchart illustrating minutiae matching process

\section{MEX-FILES}

Algorithms developed by NIST for minutiae extraction and fingerprint matching are written in $C$ programming language. Converting the source files written in $C$ language into MATLAB script is very complex and time consuming. To overcome this problem MEX-files are used. MATLAB is 
time consuming for processing large amount of data and its performance is very poor while working with images, due to slow looping functionalities. To overcome this problem it is advisable to divide the source code into different functionalities and compile the codes individually [6].

\section{A. Mex-Files Working Procedure with Example}

First build the $M E X$-file and change the path of the current directery of MATLAB for accessing the executable file. Input parameters are passed from MATLB to executable file to perform total functionality. In this example a fingerprint image is passed from MATLAB to executable file which performs image boosting operation and the output is given back to MATLAB as shown in Fig.9.

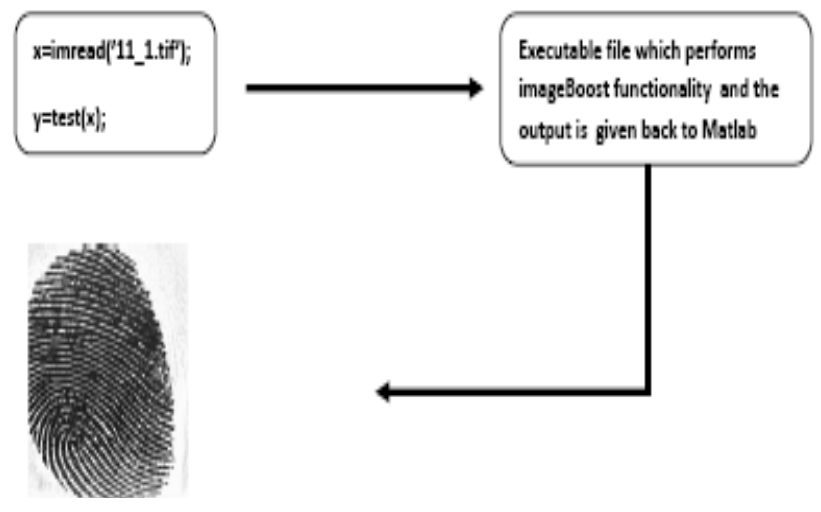

Figure 9. Flow of data between MATLAB and Visual Studio 2008 using MEX-Files

\section{RECEIVER OPERATING CHARACTERISTICS GRAPHS}

Receiver operating characteristics (ROC) is a technique used for organizing classifiers and visualizing the performance. ROC graphs are frequently used in the areas of medical decision making, machine learning and data mining. In the machine learning community the usage of ROC graphs are increased. By considering the performance of the set of classifier a graph can be drawn which remains stable at derived conditions, as the conditions of the classifier are changed the shape of the graph will also be change. So the annalist of that particular application will obtain an idea at which conditions which classifier is suitable. ROC graphs for example applied in speech/music discriminations, it may be detection of speech and detection of music [7].

"In addition to begin a generally useful performance graphical method, they have properties that make them especially useful for domains with skewed class distribution and unequal classification error cost. These characteristics have become increasingly important as research continues into the areas of cost-sensitive learning and learning in the presence of unbalanced classes" [8].

\section{A. Area under ROC Curve (AUC)}

Performance of the classifier can be depicted using ROC curve. For comparing classifiers performance, a common method is to calculate the area under the ROC curve (AUC). AUC is the position of unit squire; its value varies between 0 and 1. Random guessing creates a value between $(0,0)$ and $(1,1)$, which has a area of 0.5 . Fig.10 shows the area under ROC curves of $\mathrm{A}$ and $\mathrm{B}$. Classifier $\mathrm{A}$ has greater area compared to classifier $\mathrm{B}$, so the performance of the $\mathrm{A}$ is better compared to B performance. In Fig.5.1 classifier A is generally better than $B$ except at False Match rate $>0.25$ where B has slightly high area.

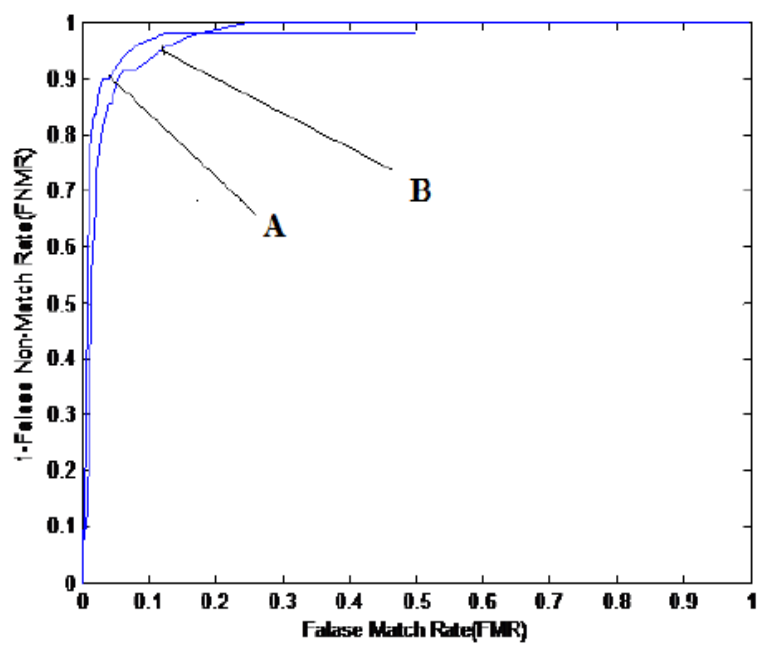

Figure 10. AUC of two classifiers A and B

\section{EXPERIMENTAL RESULTS}

FVC 2000 Database1 a [9] was used to evaluate the NBIS developed by NIST in this paper. This Database consists of 100 different users with 8 different samples from each finger. Among 100 different users top 60 users with 8 different samples from each finger are used in this work. This type of database is used to evaluate the fingerprint matching at different conditions. For plotting ROC graphs FNMR is calculated for 60 different fingers with 8 different samples from each user of genuine attempts, where a single image from the fingerprint is compared with all 8 images. FMR is calculated for 60 different fingers with 8 different samples from each finger of imposter matching in which each fingerprint is matched with all imposter images. For FNMR it will be around 3500 combinations, For FMR it will be around 67319 combinations. Scores obtained from FNMR and FMR are plotted using ROC graphs so that the performance of the NBIS can be identified. If we plot the scores obtained from different biometric systems with that curve a genuine system can be identified at different points of thresholds [2]. Results displayed below shows the ROC curve for the proposed algorithm from NIST. Fig.11 specifies $\operatorname{FMR}(\mathrm{t})$ decreases as the $t$ valuse increases and FNMR(t) increases as the $t$ value increases. The EER is the 
point at which both FMR and FNMR are identical and Fig.12 evaluates the performance of the system i.e. area under ROC curve. For evaluating the performances of two systems same plot is made for the scores obtained for different technique using same DB and the system with more AUC is treated as best system.

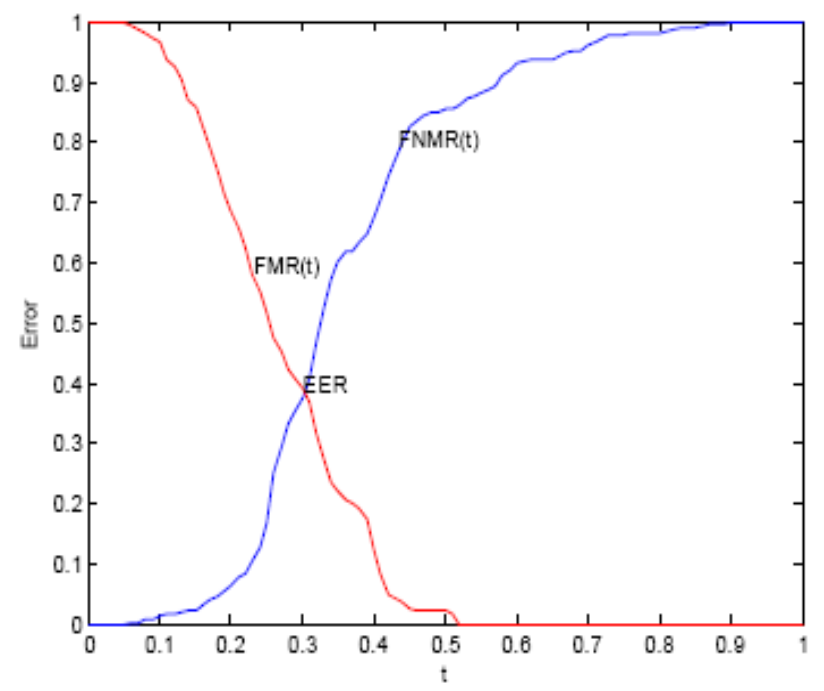

Figure 11. Plotting FMR $(t)$ and $\operatorname{FNMR}(t)$ obtained from genuine and imposter distributions (3500 genuine pairs and 67319 imposter pairs).

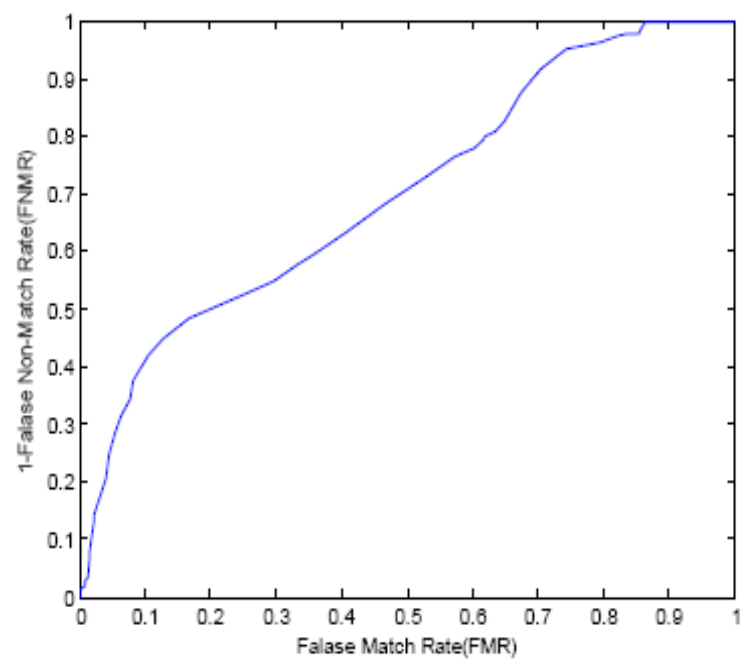

Figure 12. Plotting FMR(t) and 1-FNMR(t) obtained from genuine and imposter distributions (3500 genuine pairs and 67319 imposter pairs).

\section{CONCLUSION}

NBIS source code for fingerprint recognition is written in $A N S I C$ programming language. The NBIS is implemented with $M E X$-files routine to allow evaluation of the system in MATLAB environment. This $C$ language $M E X$-files enables to run $C$ code without reimplementing it as MATLAB script. Two $M E X$-file functions are developed with NBIS which helps to extract minutiae from grayscale fingerprint image and match two minutiae patterns to produce a match score. In this work 60 unique users with 8 fingerprints per user i.e., 480 fingerprints had been used for evaluating the NBIS. Scores obtained from minutia matcher after comparing with genuine and imposter matching had been plotted using ROC graphs. Finally the performance of the system is evaluated by analyzing ROC graphs. Results obtained by using this functionality are compared with the results obtained with other proposed technique for measuring the system performance (same DB is used in both cases).

\section{REFERENCES}

[1] Anil K. Jain, Arun Ross and Salil Prabhakar "An Introduction to Biometric Recognition," Appeared in IEEE Transactions on Circuits and Systems for Video Technology, Special Issue on Image- and Video-Based Biometrics, Vol. 14, No. 1, January 2004.

[2] D. Maltoni, D. Maio, A.K. Jain and S. Prabhakar, Handbook of Fingerprint Recognition, Springer, 2003, ISBN 0-387-95431-7.

[3] "Implementation and Evaluation of NIST Biometric Image Software for Fingerprint Recognition," Master Thesis, Blekinge Institute of Technology(in Printing).

[4] Lavanya, B.N.; Raja, K.B.; Venugopal, K.R.; Patnaik, L.M.; , "Minutiae Extraction in Fingerprint Using Gabor Filter Enhancement," Advances in Computing, Control, \& Telecommunication Technologies, 2009. ACT '09. International Conference on, vol., no., pp.54-56, 28-29 Dec. 2009.

[5] User's Guide to NIST Biometric Image Software (NBIS) available at, http://www.nis.gov/cgi-bin//get_pdf.cgi?pub_id=51097.

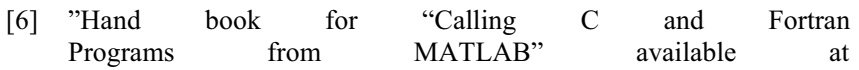
http://www.karenkopecky.net/Teaching/Cclass/MatlabCallsC.pdf.

[7] Alnadabi, M.; Johnstone, S.; , "Speech/music discrimination by detection: Assessment of time series events using ROC graphs," Systems, Signals and Devices, 2009. SSD '09. 6th International Multi-Conference on, vol., no., pp.1-5, 23-26 March 2009.

[8] Tom Fawcett,"ROC Graphs: Notes and Practical Considerations for References" , available at http://home.comcast.net/ tom.fawcett/public_html/papers/ROC101.p df,.

[9] Maio, D.; Maltoni, D.; Cappelli, R.; Wayman, J.L.; Jain, A.K.; "FVC2000: fingerprint verification competition," Pattern Analysis and Machine Intelligence, IEEE Transactions on, vol.24, no.3, pp.402-412, Mar 2002. 007.357670. 